Canadian Oncology

Nursing Journal

Revue canadienne

de soins infirmiers

en oncologie

Volume 27, Issue 2 • Spring 2017

elSSN: 2368-8076 


\section{Assessment of the feasibility and acceptability, and pre-test of the utility of an individualized survivorship care plan (ISCP) for women with endometrial cancers during the transition of the end of active treatment to cancer survivorship}

by Johanne Hébert and Lise Fillion

\begin{abstract}
The transition from the end of active treatment to survivorship holds many challenges for women with endometrial cancer (WEC) and for the organization of health services. The feasibility and acceptability of implementing an individualized survivorship care plan (ISCP) at the end of treatment are documented as potential solutions. The utility of an ISCP on three indicators (SUNS, FCRI, and HeiQ) was pre-tested by comparing two groups of WEC (control and exposed to the ISCP). The WEC exposed to the ISCP had fewer needs, a lesser intensity of fear of cancer recurrence, and better health-related empowerment skills three months after the end of treatment, as compared to the control group. Obstacles of time, resources, and organization were raised.
\end{abstract}

Background: The transition from the end of active treatment to cancer survivorship is a time of imbalance and turbulence for women with endometrial cancer (WEC). The transition to survivorship continues to be uncoordinated and the need for information about the side effects to watch for and the health risks is unmet. The implementation of an individualized survivorship care plan (ISCP) is suggested as an information and communication tool that could be a solution for facilitating the transition from the end of treatment to the beginning of survivorship.

Research objective and method: To assess and document the feasibility and acceptability of implementing an ISCP, qualitative data were gathered from WEC, oncology nurse navigators (ONN), and family doctors. A pre-experimental research design with a non-equivalent control group, an end of treatment (TO), and a three-month follow-up (T1) allowed us to pre-test its utility

\section{ABOUT THE AUTHORS}

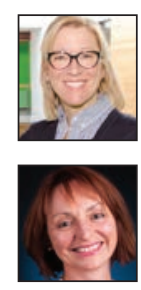

Johanne Hébert, LPN, MSc, PhD(c), Professor, Department of Nursing, Université du Québec à Rimouski (UQAR)

Lise Fillion, LPN, PhD, Full professor, Faculty of Nursing,

Université Laval

Address for correspondence: Johanne Hébert, LPN, MSc, PhD(c), Professor, Department of Nursing, Université du Québec à Rimouski, Lévis Campus, 1595 Alphonse-Desjardins Blvd., Lévis, QC G6V OA6

Phone: 418-833-8800, ext. 3243

Email: johanne_hebert@uqar.ca

https://doi.org/10.5737/23688076272153163 according to three indicators: (1) overall needs (SUNS); (2) fear of cancer recurrence (FCRI); and (3) empowerment (HeiQ) according to exposure to ISCP (control versus exposed) and to the time of measurement in the transition period (T0 versus T1).

Results: The sample was made up of 18 WEC for the group exposed to the ISCP and 13 WEC for the control group, 12 general practitioners, and two ONN. After ONN training, the ISCP completion time varied between 60 and 75 minutes, and the meeting for providing the ISCP lasted 45-60 minutes. The WEC supported the idea that meetings with the ONN and the ISCP were useful in meeting their needs for information and support. The family doctors supported its relevancy in favouring follow-up and better subsequent healthcare management, as well as in reassuring patients and avoiding a sense of abandonment at the end of treatment. Comparing the group exposed to the ISCP versus the control group, fewer reported needs can be observed: information: $35 \%$ versus $74 \%, p=.030$; professional and financial: $6 \%$ versus 19\%, $p=.057$; access and continuity: $9 \%$ versus $25 \%, p=.078$; support: $18 \%$ versus $50 \%, p=.007$, emotional: $13 \%$ versus $28 \%, p=.044)$. Moreover, at T1, empowerment according to the skill and technique acquisition sub-scale shows a higher trend ( $M=75.00$ (10.21) versus $M=64.06$ (10.67), $p=.097$ ). The level of fear of recurrence remains above the clinically significant score of 13 for both groups at the two times of measurement.

Discussion: The ISCP is an informational tool that seeks to facilitate care-related communication and coordination between specialized and primary care. It is intended to facilitate the transition from the end of treatment to survivorship and survivors' commitment to health-related empowerment behaviours. The feasibility and utility of implementing an ISCP are supported if additional professional, organizational, and financial resources are specified and mobilized.

Key words: transition, end of active treatment, cancer survivorship, individualized survivorship care plan

ynecological cancers make up $12 \%$ of new cancers in G Canadian women (Canadian Cancer Society [CCS], 2015). Endometrial cancer is the most frequent of these and is the fourth most frequent cancer in women (CCS, 2015). The number of cancer survivors continues to grow (CCS, 2015) and survivorship is recognized as a distinct phase in the continuum of care (McCabe $\&$ Jacobs, 2012). Many studies report that the transition from the end of treatment to cancer survivorship gives rise to challenges for the majority of survivors (Hewitt, Greenfield, \& Stovall, 2006) 
and for women with endometrial cancer (Jones et al., 2012; Nicolaije et al., 2012). Surviving cancer and its treatment comes with physical and psychological side effects, as well as many needs that must be taken into account (Grover et al., 2012; Hewitt et al., 2006; Salz, Oeffinger, McCabe, Layne, \& Bach, 2012).

A pivotal report by the Institute of Medicine (IOM) (Hewitt et al., 2006) reports that the transition from care to the end of treatment is often poorly coordinated and many survivors and health professionals are not aware of the long-lasting and late side effects or the heightened health risks associated with the illness and treatments. One of the report's key recommendations is to provide an individualized survivorship care plan (ISCP) to each patient finishing his or her active treatment. The ISCP is an informational tool for the survivor about the illness, treatment, long-lasting and late side effects, follow-up, resources, and health-promoting behaviours. It is also a useful tool for health professionals. The ISCP facilitates coordination and communication between the oncology team and generalists (tertiary and primary care), as well as a shared management for patient health (between the survivor, the specialized team, and the family doctor). It is recommended the ISCP be provided to the patient close to the last treatment at an in-person meeting and that a copy be sent to the family doctor (Daudt et al., 2014).

When the ISCP is provided, attention should be paid to information since this is a key factor of support (Nicolaije et al., 2012), particularly at the end of treatment (Urbaniec, Collins, Denson, $\&$ Whitford, 2011). At this phase in the continuum of care, endometrial cancer survivors have multiple needs for information (Greimel, Lahousen, Dorfer, Lambauer, \& Lang, 2011) and are dissatisfied with the information they receive (Hébert \& Fillion, 2016; Jones et al., 2012; Nicolaije et al., 2012). Providing an ISCP at the end of treatment could be an opportune moment to offer information and facilitate meeting the needs of WEC. The ISCP informs on the diagnosis, the treatments, the possible long-lasting and late side effects, and the resources available. These elements of information can reduce the ambiguity and distress caused by this health situation, as well as foster a sense of control (Arraras et al., 2010). Studies report a link between needs for information, symptoms of anxiety (Simard \& Savard, 2015; Urbaniec et al., 2011), and fear of recurrence (Brothers, Easley, Salani, \& Andersen, 2013; Greimel et al., 2011; Jones et al., 2012; Salani, 2013) in cancer survivors. Providing an ISCP could reduce uncertainty and have an impact on the fear of recurrence often reported by WEC, thus contributing to a better management of distress and to a sense of control (Faul et al., 2012). It could also be a strategy to favour empowerment behaviours by encouraging the survivors' active involvement in their health. More particularly, the ISCP fosters empowerment by inviting survivors to ask questions of their healthcare team during follow-up appointments (Faul et al., 2012; Watson, Sugden, \& Rose, 2010), by raising awareness about the importance of adopting health-promoting behaviours after the end of treatment (Sprague et al., 2013), and by specifying an action plan to be implemented according to specific directions (McCorkle et al., 2011). Empowerment consists of one's ability to manage one's own state of health, the possibility of collaborating with the healthcare team, and accessing quality support care (McCorkle et al., 2011)
Moreover, the implementation of an ISCP could improve coordination of care and communication between caregivers in this phase of the care trajectory (Hewitt et al., 2006; HillKayser et al., 2013; Jefford et al., 2011).

However, it is reported that few survivors have access to an ISCP (Rechis, Beckjord, \& Nutt, 2014; Sabatino et al., 2013) and that only $10 \%$ of gynecological cancer survivors may benefit from such a plan (Brothers et al., 2013; Grover et al., 2012; Sabatino et al., 2013; Salani, 2013).

Studies have documented the development of an ISCP for women with breast cancer (Clausen et al., 2012; Dulko et al., 2013; Smith, Singh-Carlson, Downie, Payeur, \& Wai, 2011), for survivors of colorectal cancer (Baravelli et al., 2009; Salz et al., 2012), and for other types of cancer (Mayer, Gerstel, Leak, \& Smith, 2012) based on existing ISCPs. Some recommend personalizing the ISCP to better meet the complex needs of survivors (Haq et al., 2013). It is also recommended to develop these plans in collaboration with the multidisciplinary team and oncology outreach managers so that the care processes and the organization's resources be considered (Jefford et al., 2011; Mayer et al., 2014). To our knowledge, a study has yet to document the development of an ISCP that considers the needs of endometrial cancer survivors and those of the multidisciplinary team and the oncology outreach manager, as well as the context and the available organizational resources.

Taking these recommendations into account, a personalized ISCP for women with endometrial cancer (WEC) was first developed in collaboration with WEC and their multidisciplinary specialized care teams in consideration of organizational resources as the first phase of the doctoral research of the primary author (Hébert \& Fillion, 2016). The second phase, presented here, aimed to assess the feasibility and acceptability of the ISCP, and to pre-test its utility for WEC on three indicators retained for this study (needs, distress/fear of recurrence, and empowerment). It also aimed to document certain aspects of the ISCP's feasibility and its acceptability by general practitioners as well as to have the ONN of the targeted clinics implement it.

\section{METHODOLOGY}

\section{Design and Objectives}

The research was based on a sequential exploratory design (Creswell \& Plano Clark, 2011). This design included a first development phase that was the development and validation of the ISCP (Hébert \& Fillion, 2016). The second phase was an exploration of the implementation, and was made up of three objectives: (1) to document the feasibility of implementing an ISCP; (2) to assess the acceptability of the ISCP; and (3) to pretest its utility in meeting overall needs, reducing distress (fear of recurrence), and fostering empowerment.

Qualitative and quantitative data were gathered from WEC to meet these objectives. To meet the first and second objective, an individual interview, guided by the study's concepts and variables, was carried out with two oncology nurse navigators (ONN) from the oncology team involved in implementing the ISCP. For the second objective, a questionnaire, accompanied by a cover letter presenting the study and a copy of the ISCP, was mailed to the family doctors of study participants. To meet the third objective, a pre-experimental research design with a non-equivalent control group and two times of 
measurement $(\mathrm{T} 0=$ end of treatment and $\mathrm{T} 1=$ three-month follow-up) were used. The two groups of WEC (exposed versus control) were compared for the three indicators: overall needs, fear of recurrence, and empowerment.

\section{Participants and Procedure}

For qualitative data collection from WEC and ONN, semi-structured guided interviews were conducted with discussion guides developed from the study's conceptual models (Meleis et al., 2000; Fitch, 1994). Allowing us to gather family doctors' perceptions of the relevancy and utility of the ISCP, this questionnaire was accompanied by a cover letter presenting the study and a copy of the ISCP, and was mailed to all the general practitioners who had a patient participating in the study. To compare the WEC according to conditions of exposure, two intentional samples were constituted according to the following inclusion criteria: (1) having endometrial cancer and having received adjuvant treatment (chemotherapy, radiation therapy, or brachytherapy) alone or combined; (2) having finished active cancer treatment; (3) having a family doctor; and (4) being 18 years of age or older. The two groups frequented the same specialized care team. The first—-the control group-was made up of WEC treated by this team before the introduction of the ISCP. The second — the exposed group—was made up of WEC treated by this same team after the ISCP was introduced. The recruitment procedure was similar for the two groups. The team's two ONN specialized in gynecological cancers and a nurse manager identified eligible patients and informed them of the study. Those who were interested in receiving more information were invited to meet the research student who informed them of the protocol and invited them to take part in the study. Those who accepted to participate in the study filled out a consent form. The research procedure differed, however, according to the conditions of exposure. The study received the ethical approval of the university hospital centre in which it took place.

\section{Conditions of Exposure}

Two conditions of exposure were retained to pre-test the utility of the ISCP in meeting the three indicators (overall needs, fear of recurrence, and empowerment) measured in WEC.

The first condition was providing the ISCP (or the nursing intervention). This was performed by the ONN at the last treatment. During this meeting, information about all the elements contained in the ISCP was shared and WEC's concerns about post-treatment were discussed. The information that had to be systematically covered when the ISCP was provided to the patient was: symptoms to monitor; follow-up with the oncologist and generalist; the resources available; and behaviours of empowerment during the survivorship period. The person-centred approach was retained (Street, Makoul, Arora, \& Epstein, 2009) to provide the information according to the needs the WEC expressed. The second condition was the transition period, which corresponded with the two measurements (end of treatment [T0] and the systematic follow-up three months post-treatment [T1]).

For the exposed group, the first measurement occurred when the ISCP was provided and the ONN distributed the study questionnaires (SUNS, FCRI, and HeiQ). The second measurement was conducted during the three-month post-treatment follow-up appointment in an interview with the research student. At both measurements, the questionnaires were submitted to participants in self-addressed stamped envelopes addressed to the research student.

For the control group, the sample was created in the Phase 1 of the doctoral research (Hébert \& Fillion, 2016), carried out before the ISCP was introduced and with the same oncology team. A meeting was scheduled with the research student at the end of treatment (T0) or at the three-month follow-up point (T1). The interview looked specifically at the needs experienced during this transition period. The same questionnaires as those submitted to this study's exposedgroup participants were submitted in self-addressed stamped envelopes addressed to the research student. No ISCP was provided to the participants in this group and no meeting was systematically held with the ONN at the end of treatment.

\section{Data Collection}

Three instruments measured the three retained indicators (overall needs, fear of recurrence, and empowerment). The overall needs were measured by the Survivors Unmet Needs Survey (SUNS) (Campbell et al., 2011). This measure includes 89 items divided into five sub-scales of needs. The short Frenchlanguage version used in this study was made up of 28 items (Propel Centre for Population Health Impact). Each of these items was assessed on a Likert scale from 0 ("All my needs were met") to 4 ("Almost none of my needs were met"). A high score indicates that needs were not met. Fear of recurrence was measured by the Fear of Cancer Recurrence Inventory (FCRI) (Simard \& Savard, 2009). This questionnaire includes 42 items divided into seven sub-scales. In this study, the sub-scale of severity, which contains nine items, was used. Each item is assessed on a Likert scale from 0 ("not at all") to 4 ("very much"). The possible scope of the score varies between 0 and 36. A high score indicates severe fear of recurrence. For this sub-scale of severity, a score of 13 and above is clinically significant and shows a level of fear of recurrence for which further assessment is recommended (Simard \& Savard, 2015); this sub-scale presents a satisfactory loyalty index ( $\alpha$ de Cronbach $=0.89$ ). Empowerment was measured by the Health Education Impact Questionnaire (HeiQ) (Osborne, Elsworth, \& Whitfield, 2007). This study used a version by Maunsell et al. (20I4), which measures empowerment and was made up of five of the original measure's sub-scales. The French-language version (Canada) was validated for people with cancer (Brunet et al., 2015). Each item was assessed on a Likert scale from 1 ("strongly disagree") to 4 ("strongly agree"). A high score signifies a high level of empowerment behaviours. All scales were converted on a 0-100 scale. The instrument shows a satisfactory loyalty index ( $\alpha$ varies between 0.87 and 0.94 according to sub-scale).

For the evaluation of the acceptability and feasibility with WEC of the exposed group, discussion guides were developed based on the central concepts of the theoretical models retained for the doctoral study. These included Meleis, Sawyer, Im, Hilfinger Messias, and Schumacher's (2000) theory of transition, the supportive care framework in oncology (Fitch, 1994) and the feasibility study criteria put forth by 
Bowen et al. (2009). Certain other aspects of Feinstein's clinical tool assessment framework (1987) were also used.

For the interviews with the ONN, a discussion guide was developed according to the concepts and considerations proposed by Bowen et al. (2009) as feasibility study criteria, and by Feinstein (1987) for the development of clinical tools. These same concepts and considerations guided the development of the questionnaire to collect the perceptions of family doctors. This questionnaire had six open questions on the relevancy, utility, and content of the ISCP, and space for comments. These frameworks and the concepts guiding the development of the discussion guides and the questionnaires for family doctors are presented in Table 1.

\section{Data Analysis Plan}

These descriptive statistical data were carried out to describe WEC's socio-demographic and clinical characteristics (demographic and medical variables) according to each group (control or exposed). For the feasibility and acceptability with WEC and ONN (objectives 1 and 2), the analysis of qualitative data was carried out according to the Miles and Huberman (2003) approach. Individual interviews were self-recorded in digital format and transcribed in full. The data were organized and coded using the NVivo 10 software. Qualitative data generated by the questionnaires for family doctors were also analyzed according to a thematic approach. The emerging categories were grouped by the themes and reported in percentage of occurrences.

Table 1: Framework and concepts retained to guide the collection, analysis, and interpretation of qualitative data

Conceptual frameworks

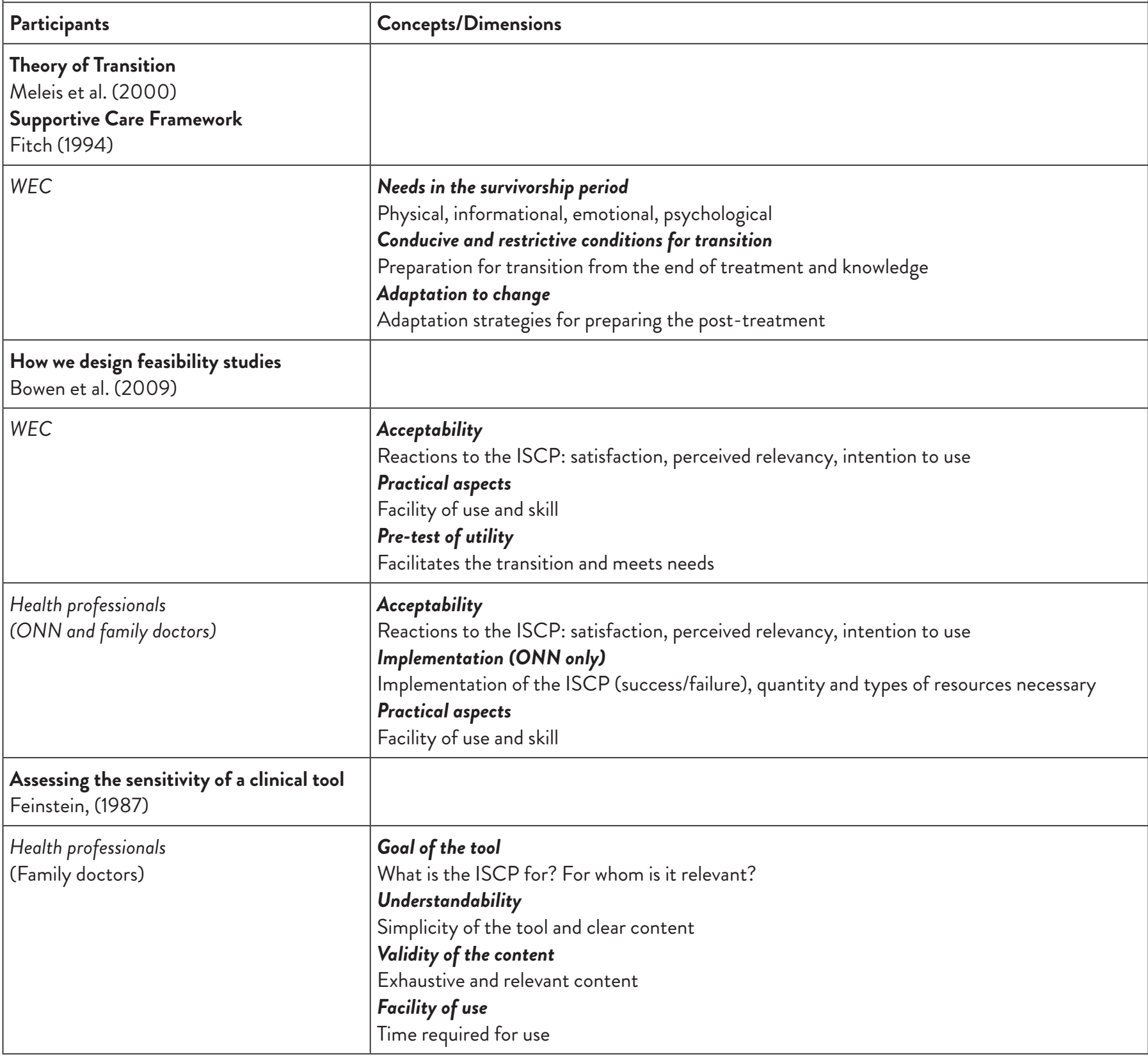


For the third objective, the statistical analyses made it possible to compare the averages obtained for each indicator retained (overall needs, fear of recurrence, or empowerment) according to group (exposed or control) and transition period (end of treatment [T0] or three-month follow-up [T1]). Because of the small size of the study sample, a permutation test was used to compare the two groups at each measurement using the NPAR1WAY procedure in the SAS/STAT software (2013). The comparison was limited to the exploration of possible connections between the conditions of exposure to the ISCP and the retained indicators.

\section{RESULTS}

In this section, the characteristics of the WEC sample will be described. The analysis results documenting the feasibility of ONN introducing an ISCP will then be presented. The results describing the acceptability and certain aspects of the feasibility with WEC, ONN and family doctors will be explored. Finally, the pre-test results on the three indicators in regards to the ISCP's utility will end this section.

\section{Sample of WEC and Health Professionals}

The control group was made up of nine WEC at T0 and four WEC at T1. Twenty-seven WEC accepted to participate in the study to create the ISCP-exposed group. An ISCP was completed by the ONN for each participant. Of this number, three participants refused the meeting at which the ISCP was provided, two had to extend their treatments, one stopped her treatment, and one ended her treatment sooner than planned and was, therefore, no longer available for a meeting. In all, 20 ISCP were provided to WEC at the end of their treatment. Of this number, 18 had completed the questionnaires provided to them during the meeting with the ONN (T0) and 12 had done so for the three-month follow-up (T1). These latter were also met with for an individual interview. The sociodemographic and medical characteristics of the two groups are presented in Table 2. Results suggest that for age, $(t=.95)$, education $(t=1.0)$, and employment $\left(\mathrm{X}^{2}=.85\right)$, the two groups were not significantly different. In regards to the treatments received, Fisher's exact test reports that the two groups are not significantly different for chemotherapy $(\mathrm{p}=.094)$ and radiation therapy $(\mathrm{p}=.164)$. However, the proportion of WEC who received brachytherapy was higher in the exposed group $(\mathrm{p}<.0001)$.

For the family physicians, 18 questionnaires were sent by mail and 12 were returned to the research student, thus corresponding to a $67 \%$ response rate.

\section{Feasibility of Implementing an ISCP at the End of Treatment}

The appropriation period of the ISCP required a total of six meetings between the ONN and the research student. Each section of the ISCP was reviewed and dropdown menus with multiple-choice answers were created to simplify its completion and use. The information to cover in keeping with a person-centred approach were discussed in the goal of better meeting the needs WEC express. Moreover, the tasks of adding to clinical activities the inclusion of the ISCP in patient files and the coordination of the end-of-treatment meeting during which WEC received their ISCP was clarified.

\begin{tabular}{|c|c|c|}
\hline Characteristic & $\begin{array}{c}\text { Control } \\
\text { group } N=13\end{array}$ & $\begin{array}{c}\text { Exposed } \\
\text { group } N=18\end{array}$ \\
\hline \multicolumn{3}{|l|}{ Age } \\
\hline $44-60$ years & 46.2 & 44.4 \\
\hline 61 years and + & 53.8 & 55.6 \\
\hline \multicolumn{3}{|l|}{ Marital status } \\
\hline Single & 16.6 & 29.4 \\
\hline Married & 44.4 & 52.9 \\
\hline Common-law spouse & 22.2 & 5.8 \\
\hline Separated & & 5.8 \\
\hline Divorced & & 5.8 \\
\hline Widowed & 16.6 & \\
\hline \multicolumn{3}{|l|}{ Level of education } \\
\hline Elementary & 8.33 & \\
\hline Secondary & 25.0 & 47.1 \\
\hline Professional diploma & 16.7 & 5.9 \\
\hline CEGEP/College & 8.33 & 5.9 \\
\hline University & 41.6 & 41.2 \\
\hline \multicolumn{3}{|l|}{ Employment status } \\
\hline Full time & 8.33 & 17.6 \\
\hline Part time & & 17.6 \\
\hline Unemployed & & 5.9 \\
\hline Illness & 25.0 & 17.6 \\
\hline Retired & 66.7 & 41.2 \\
\hline Family doctor & 91.7 & 83.3 \\
\hline \multicolumn{3}{|l|}{ Treatments received } \\
\hline Chemotherapy only & 25.0 & 5.6 \\
\hline $\begin{array}{l}\text { Chemotherapy and radiation } \\
\text { therapy }\end{array}$ & 8.33 & \\
\hline $\begin{array}{l}\text { Chemotherapy and } \\
\text { brachytherapy }\end{array}$ & 8.33 & 22.2 \\
\hline $\begin{array}{l}\text { Radiation therapy and } \\
\text { brachytherapy }\end{array}$ & 16.7 & 11.1 \\
\hline Brachytherapy only & 8.33 & 22.2 \\
\hline $\begin{array}{l}\text { Chemotherapy/radiation } \\
\text { therapy/brachytherapy }\end{array}$ & 33.3 & 38.9 \\
\hline
\end{tabular}


After the appropriation period, the procedure for completing the ISCP by a research nurse (a newly retired nurse navigator from this clinic) was implemented. Since the study of the ISCP came at the same time as the gradual introduction of electronic patient files to the clinical setting, the ISCP was completed with data taken from both the paper and the electronic files of each WEC. Finding some information required considerable time (e.g., chemotherapy specifics). Certain information was not in the files (e.g., dose of brachytherapy or radiation therapy specifics). The time required to find this information and complete the ISCP varied between 60 and 75 minutes per participant.

The length of the meeting in which the ONN provided the WEC with the ISCP varied between 45 and 60 minutes. The ONN did not make negative comments about the addition of this clinical task. However, making the appointment and coordinating the meeting were an additional and less-appreciated task. Without IT tools to coordinate patient care trajectory appointments, the ONN were required to make considerable effort to coordinate this task. Before introducing the ISCP, no systematic end-of-treatment meeting was planned in the care trajectory. The main coordination obstacle was caused by unforeseen changes in the date or hour of last treatments (e.g., brachytherapy). Another difficulty was around scheduling conflicts if two WEC finished their last treatment at the same time (same day and hour). For this reason, three of the ISCP developed for recruited participants were never provided.

In sum, providing an ISCP required additional time to prepare, complete, and coordinate, as did the scheduling of an additional meeting otherwise absent from the care trajectory. In part stemming from an organization unprepared for additional follow-up, this time and additional effort was assessed by the ONN as a major hindrance to integrating this intervention into their regular practice. Without an additional resource to complete the ISCP and modify the care trajectory to include an end-of-treatment meeting, integrating the ISCP would appear to be unrealistic and, therefore, in the current context, the feasibility of introducing an ISCP is limited.

\section{Acceptability of the ISCP}

Despite this issue of feasibility, the ONN supported the added-value of an ISCP for the WEC at the end of active treatment. They mentioned that participants expressed feelings of reassurance to be able to ask questions and share concerns regarding the post-treatment period in a formal meeting foreseen for this purpose. The ONN pointed out that discussion of the ISCP content made it possible to clarify several elements regarding monitoring and follow-up (symptoms, follow-up with the specialist and the generalist, resources, and health-promoting behaviours). Moreover, the ONN appreciated being able to discuss the behaviours of empowerment and the importance of check-ups with the family doctor in the survivorship phase. Providing an ISCP was acceptable, even desirable. However, current organizational conditions make it impossible.

To overcome the obstacle of feasibility of integrating this intervention into their practice, ONN suggested several potential solutions. The time allotted to the appropriation of the ISCP was deemed necessary. Training would, therefore, be needed when new ONN join the team. The ONN also suggested each professional involved in the care trajectory complete the appropriate section (e.g., that the hematologist-oncologist fills out the chemotherapy section [medications, cycles, and specifics]). The acceptability of this procedure with the actors involved would, however, require documentation with the multidisciplinary team. The ONN also suggested that the medical data contained in the ISCP could be completed incrementally. In their opinion, the ISCP could be useful for patients in understanding their medical situations and for the ONN when completing the ISCP. Finally, the end-of-treatment meeting should be systematically integrated in the patient care trajectory to facilitate coordination and patient participation. In sum, like the ISCP feasibility, the ONN feel organizational limitations compromise the acceptability of the ISCP.

Echoing the ONN, WEC reported that the meeting with the ONN, during which the ISCP was provided, was useful for receiving relevant information regarding the illness, the treatments received, and the post-treatment period (knowing which symptoms to watch for, subsequent medical follow-up, and health-promoting behaviours). They also reported the relevancy for better understanding their situation: "I had time to ask all my questions" (WEC 4) and "I received all the information I needed" (WEC 1). Several participants mentioned that the meeting with the ONN helped them better understand the scope of the illness and the treatments they had received.

For the participants, this end-of-treatment meeting also helped them realize that the ONN could continue to be a resource available to them in the post-treatment period and that this would facilitate their transition to survivorship: "She explained to me that even after treatment was over, I could contact her if I had a problem" (WEC 6). In a similar way facilitating this transition, the participants also reported having consulted the ISCP to validate their physical symptoms (pain, lymphedema, intestinal problems) to learn what follow-up was forthcoming, and remember which resources were available. Others mentioned that the information contained in the ISCP would be useful for their family doctors or other health professionals who don't have their file: "I was glad to see the information that was there... it is important for the doctor who is going to see me" (WEC 3). However, three participants mentioned that they did not use the ISCP after their meetings with the ONN.

Three themes emerge from analysis of family doctors' answers to the open questions regarding their perceptions of the acceptability of the ISCP. These are presented mostly in percentages. The first was in regard to the relevancy for WEC. All the physicians $(100 \%)$ support that implementing the ISCP is pertinent for WEC. Some added that the ISCP would favour follow-up and better overall patient care, due to reassurance and avoiding patients feeling abandoned at the end of treatment.

The second theme was in regards to the utility of the ISCP for general physicians. Many (92\%) felt that the ISCP would be useful to them in better understanding the medical follow-up 
their patients required. Further information would nevertheless be desirable for certain participants regarding the complexity of the follow-up when several comorbidities were present and regarding the importance in these cases that the responsibility for follow-up be shared with the oncology team in the first year post-treatment.

The third theme looked at the impact of the ISCP on the continuum of care. Respondents $(83 \%)$ mentioned that the ISCP could facilitate the continuity of care, as well as of relationships between the patient and the family doctor, while $75 \%$ thought that it could better meet the emotional and informational needs of WEC. Sixty-seven percent (67\%) specified that it could facilitate WEC's transition at the end of treatment. Moreover, $83 \%$ of the generalist doctors surveyed reported that the ISCP would be transferable to other cancers, such as breast, prostate, and colorectal. In general $(83 \%)$, the respondents found the content to be adequately developed, clear, precise, and complete. Only one participant felt that the suggestion of symptoms (possible side effects and symptoms to which a doctor should be alerted) could contribute to increasing patient anxiety. In sum, all perceived the ISCP as acceptable, even desirable.

\section{Utility of the ISCP}

Comparisons between the control and exposed groups, as well as the comparisons between the times of measurement (at end of treatment and at the three-month follow-up point) are presented by category of indicators and summarized in Table 3.

Needs indicators (SUNS): At T0, no significant difference in needs was observed between the two groups. However, at T1, results indicate fewer needs in the group with an ISCP. The averages observed in the needs at $\mathrm{T} 1$ for this group are lower on all sub-scales: (1) information: $35 \%$ of the exposed group versus $74 \%$ of the control group had unmet needs, $\mathrm{p}=.030$; (2) professional and financial: $6 \%$ with an ISCP versus $19 \%$ control, $\mathrm{p}=.057$; (3) access and continuity: $9 \%$ with

Table 3: Variations in the indicators according to group (control versus exposed) at each time of measurement (T0 and T1)

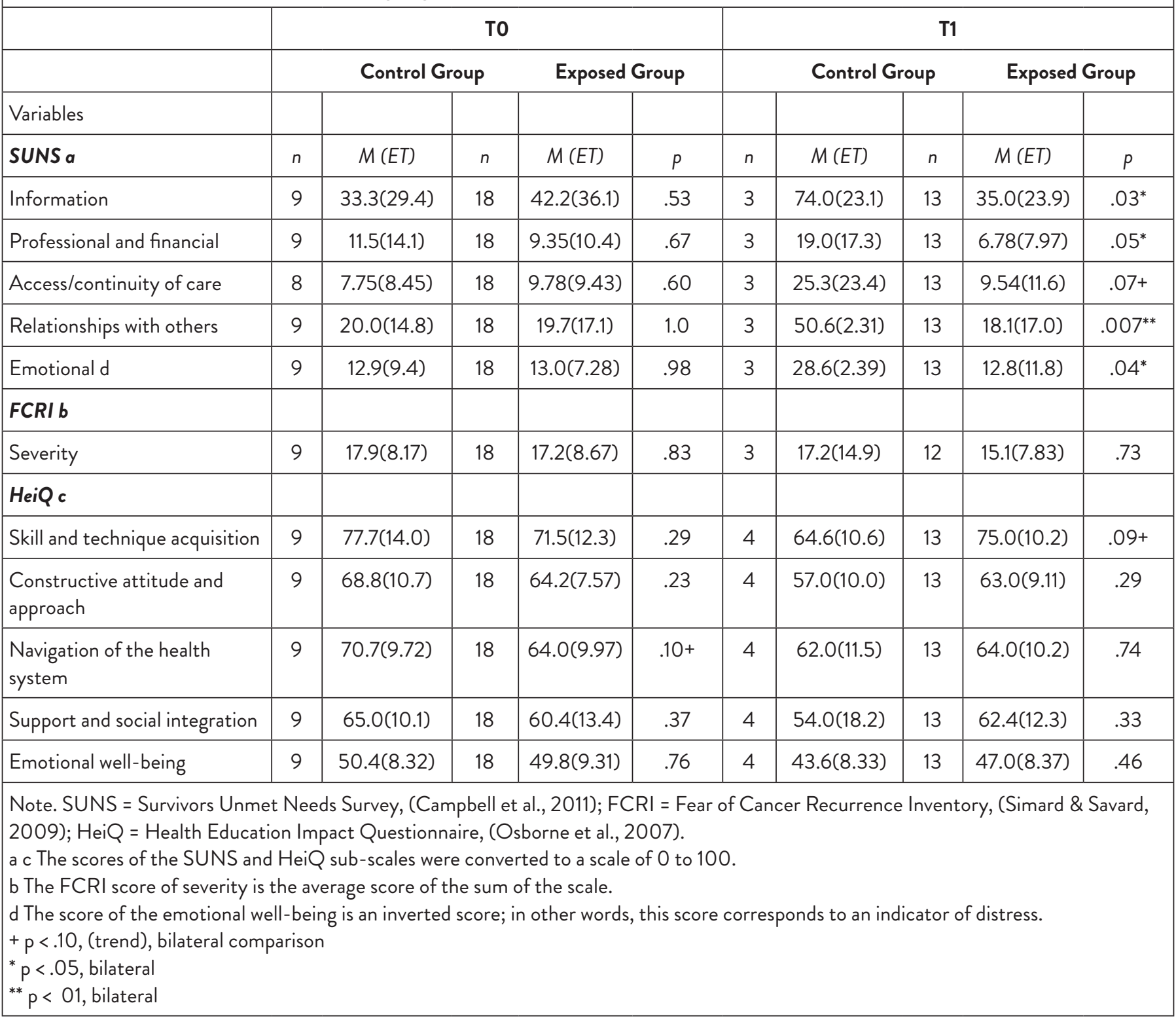


ISCP versus $25 \%$ control, $\mathrm{p}=.078$; (4) relational (support): $18 \%$ with ISCP versus $50 \%$ control, $p=.007$, and; emotional: $13 \%$ with ISCP versus $28 \%$ control, $\mathrm{p}=.044$.

FCRI: The average score on the severity index for the fear of recurrence between T0 and T1 dropped for the exposed group (17.22 versus 15.12), compared to the control group, which remained comparable (17.97 versus 17.28$)$ although no difference between the groups was significant. Moreover, the results suggest that the level of fear of recurrence remains above the clinically significant level of 13 and more for the two groups at both times of measurement. Specifically, $66 \%$ of the participants in the control group presented a clinically significant score of 13 and above at both measurements and for the exposed group, $55 \%$ at T0 and $42 \%$ at T1.

HeiQ: Overall, the scores of the sub-scales of empowerment were observed to be higher in the control group at T0 than in the exposed group. The opposite is observed, however, at T1 where the scores on the sub-scales of empowerment seem higher in the exposed group than in the control group. No difference was significant, except at T1, where the exposed group presents a trend towards a higher score than the control group on the skill and technique acquisition sub-scale 75.00 (10.21) versus 64.06 (10.67), $\mathrm{p}=.097$.

In sum, no difference was noted between the groups at T0 for any indicator. At T1, fewer needs were observed for four of the five categories, as well as a trend towards a better score of empowerment on one of the five sub-scales for the exposed group when compared to the control group. The fear of recurrence remains clinically significant for the two groups at both times of measurement.

\section{DISCUSSION}

The study sought to pre-test an individualized follow-up care plan (ISCP) for women with endometrial cancer (WEC) that was developed in a first research phase. The objectives were to document the feasibility and acceptability, and pre-test the utility of the ISCP in meeting overall needs, reducing distress (fear of recurrence), and fostering empowerment at end of active treatment.

In terms of feasibility, the additional time or resources dedicated to appropriating the ISCP (content and computerized format) and its completion was perceived as necessary. The time required to find information and complete the ISCP was, however, comparable to that reported in many studies (Curcio, Lambe, Schneider, \& Khan, 2012; Dulko et al., 2013; Stricker et al., 2011). The length of the meeting during which the ISCP was provided was also similar to that of other studies (Curcio et al., 2012; Stricker et al., 2011), although one study reported a shorter time (between 15 and 30 minutes; Salz et al., 2014). Making appointments and coordinating the ISCP at the last treatment session also required additional time and effort, and was sometimes perceived as being difficult. Despite results that were comparable to other studies for completing the ISCP and providing it to patients, this additional time added to the ONN's clinical tasks and, therefore, compromises the feasibility of implementing an ISCP under current conditions of service organization.
In terms of acceptability, WEC felt the meeting with the ONN during which they received an ISCP was useful and noted that it prompted discussion of the information contained in the ISCP and addressed their post-treatment concerns. The ONN was perceived as an appreciated resource for the post-treatment period, as was the possibility of medical follow-up by the family doctor facilitated by the ISCP. Participants reported an improvement in knowledge about the symptoms to watch for, the follow-up to come and the resources available to them. Results are comparable to those of other studies on various types of cancer that underscore the importance of survivors receiving information regarding their diagnosis, treatments, recommended follow-up care, and care offered by the oncology team (Salz et al., 2014) in a condensed written format (Faul et al., 2012). The ISCP meets these needs for information and improves knowledge of the illness and treatments (Curcio et al., 2012; Faul et al., 2012). According to Sprague et al. (2013), survivors are satisfied with the ISCP and find it to be useful and to facilitate an understanding of their diagnoses, treatments, follow-up, and the recommended health-promoting behaviour. Feeling informed would seem to help in planning changes in behaviour and foster responsibility for one's health (Hill-Kayser et al., 2013). Moreover, survivors report that it improves communication with the oncology team and other health professionals (Faul et al., 20I2).

Our study highlights how the ISCP facilitates the monitoring of lasting symptoms and the transfer of information to the family doctor. The ONN and general physicians surveyed in this study point out the relevancy of the ISCP, saying that it would help meet needs for information and favour the feelings of reassurance that facilitate the transition to survivorship. It would also facilitate the continuity of care and communication between health professionals. Several studies highlight that a majority of health professionals approve the concept, format, and structure of the ISCP (Faul et al., 2012; Forsythe et al., 2013) and believe in the importance of patients receiving information on the content provided in the ISCP (Faul et al., 2012; Salz et al., 2014). Moreover, the ISCP could improve both the coordination and the continuum of care (Faul et al. 2012; Forsythe et al., 2013), it would improve communication between health professionals (Faul et al., 2012; Forsythe et al., 2013; Hill-Kayser et al., 2013), and it would be useful for family doctors in monitoring symptoms and knowledge of follow-up (Curcio et al., 2012; Forsythe et al., 2013; Salz et al., 2014). However, although all participants found the end-of-care meeting with the ONN useful, three among them mentioned not having used the ISCP thereafter. Despite the ONN's perception of acceptability of the ISCP, the time required for its appropriation, completion, and coordination of the end-of-treatment appointment limits its feasibility in the clinical setting.

As for the utility of the ISCP in meeting overall needs, reducing emotional distress (fear of recurrence), and favouring empowerment, our results, like those of Jefford et al. (2011), suggest that the ISCP would help reduce needs three months after the end of treatment. In regards to fear of recurrence, although the average score seems to improve for participants with an ISCP, their level of fear of recurrence remains 
high and more extensive investigation would be recommended for the majority of WEC. Other studies highlight how the ISCP encourages patients to ask their medical team questions about the treatments received and the symptoms to monitor, and its impact on the fear of recurrence by bringing "peace of mind" (Faul et al., 20I2). Although our study shows a slight improvement for the group with an ISCP, 55\% continued to have a clinical level of fear of recurrence of 13 and above at the end of treatment; and three months post-treatment, $42 \%$ had this fear, which is shown to increase the risk of developing anxiety (Simard \& Savard, 2015). The screening for distress in this critical period in the trajectory would be useful for specifying the needs for support and optimizing support care (Fillion et al., 2011). Regarding the effects of the ISCP on empowerment, a trend to improvement in behaviours of empowerment between the group with an ISCP and the control group three months post-treatment was observed. In sum, despite the limitations inherent to the research design, a positive impact needs seems to be observed, although it does not have a repercussion on distress or behaviours of empowerment. Screening and management of distress, as well as intervention on empowerment (i.e., a reinforcement of one's power to take action), may need to be improved to facilitate transition to survivorship. Moreover, ONN appreciated the time dedicated to this aspect of their work. Systematic screening of distress at the end of treatment and a reinforcement of therapeutic strategies of empowerment could be a complementary measure at the end-of-treatment meeting when the ISCP is provided.

Although a majority of health professionals support the implementation of the ISCP and suggest it become a standard in care, others question its added-value for the quality of follow-up care (Forsythe et al., 2013; Salz et al., 2014). In the United States, the ISCP did, however, become a standard in care for cancer programs in 2015 (American College of Surgeons, 2012). To favour the implementation of the ISCP, several professional organizations (ASCO, LIVESTRONG, Journey Forward) have developed models that include recommendations by the IOM (2006) and are available online (Stricker \& O’Brien, 2014). Canadian recommendations suggest the organization of services in cancer survivorship based on best practices for optimizing survivor health and well-being at the end of active treatment (Howell et al., 2011). Survivors must have access to coordinated and multidisciplinary support services to meet the wide range of needs at the end of active treatment to prolonged survivorship. The IOM report suggests an individualized follow-up care plan be offered to all patients ending active treatment (including IOM recommendations, 2006) by a designated member of the healthcare team. It also suggests an individual meeting at the end of treatment with the person and his or her loved ones to prepare follow-up care and minimize distress in the transition from the end of active care to the survivorship phase in the continuum of care. Our study casts light on several gaps in current practice and presents interesting avenues for transferring these recommendations to practice. However, it encounters the major barrier of feasibility. Although the ONN are well-positioned and motivated to integrate the ISCP into their practice and perceive the acceptability and utility of an ISCP for WEC, they doubt the feasibility of being able to integrate it into their practice without an additional resource to complete the ISCP and a desire to systematically include an end-of-treatment meeting to the care trajectory.

\section{Limitations}

This study on the feasibility of integrating an ISCP into practice has several limitations. Unlike the development of an ISCP, in which all the actors' points of view were taken into consideration, in this second phase of assessment, the feasibility was only documented based on the ONN involved in the oncology team. An assessment of feasibility by other team members and by the resource managers would have been desirable. What's more, the small number of study participants and the exploratory research design with a non-equivalent group considerably limits comparison of the groups and documentation of the utility. Nevertheless, the results do show a profile of improvement in terms of information that is in keeping with that reported by previous studies. The qualitative aspect documents the feasibility and acceptability of implementing an ISCP, as recommended in the literature.

\section{CONCLUSION}

This preliminary study supports the added value of the ISCP for survivors of endometrial cancer during the transition from the end of treatment to survivorship to meet needs for information and favour communication between survivors and health professionals. Although survivors reported an overall high level of satisfaction with the ISCP in other studies (Brennan et al., 2014; Klemanski et al., 2015; Stricker et al., 2011), a lack of measurable proof of its added value has also been found (Brennan et al., 2014; Klemanski et al., 2015; Mayer et al., 2014) and the best practices have yet to be validated by research (Stricker \& O’Brien, 2014). Despite growing support for ISCP, it is slow to be adopted. The reasons for this include accessibility to electronic files, limited available resources, the lack of remuneration, and the lack of time to complete the ISCP (Rechis et al., 2014; Stricker \& O'Brien, 2014), as well as a resulting increase in nursing interventions to improve management of distress and empowerment. IOM recommendations (2006) pose a sizeable challenge for health professionals since there is no consensus on the characteristics of the ISCP or how to make it operational in the clinical setting (Brennan et al., 2014; Daudt et al., 2014; Keesing et al., 2015). Recent studies show a considerable variation in approaches for the development, implementation, and evaluation of the ISCP, and few survivors receive an ISCP (Keesing et al., 2015; Stricker et al., 2011). This study provides concrete avenues. It specifies the importance of inserting an ISCP into a broader program (integrating it into the survivorship phase in the cancer trajectory and favouring not only information about, but also screening for distress) and integrating empowerment into cancer survivorship.

\section{CONFLICTS OF INTEREST}

The authors have no potential conflicts of interest. 


\section{REFERENCES}

American College of Surgeons. (2012). Cancer Program Standards: Ensuring patient-centered care. Chicago, IL: Commission on cancer.

Arraras, J.I., Greimel, E., Sezer, O., Chie, W-C., Bergenmar, M., Costantini, A., Young, T., Vlasic, K.K., \& Velikova, G. (2010). An international validation study of the EORTC QLQ-INFO25 questionnaire: An instrument to assess the information given to cancer patients. European Journal of Cancer, 46(15), 2726-2738. doi:10.1016/j.ejca.201006118

Baravelli, C., Krishnasamy, M., Pezaro, C., Schofield, P., LotfiJam, K., Rogers, M., Milne, D., ... Jefford, M. (2009). The views of bowel cancer survivors and health care professionals regarding survivorship care plans and post treatment follow up. Journal of Cancer Survivorship: Research and Practice, 3(2), 99-108. doi:10.1007/ s1176400900861

Bowen, D.J., Kreuter, M., Spring, B., Cofta-Woerpel, L., Linnan, L., Weiner, D., Bakken, S.,[... Fernandez, M. (2009). How we design feasibility studies. American Journal of Preventive Medicine, 36(5), 452-457. doi:10.1016/j.amepre.2009.02.002

Brennan, M.E., Gormally, J.F., Butow, P., Boyle, F.M., \& Spillane, A.J. (2014). Survivorship care plans: a systematic review of care plan outcomes. British Journal of Cancer, 111(10), 1899-1908.

Brothers, B.M., Easley, A., Salani, R., \& Andersen, B.L. (2013). Do survivorship care plan impact patients' evaluations of care? A randomized evaluation with gynecologic oncology patients. Gynecologic Oncology, 129(3), 554-558.

Brunet, J., Lauzier, S., Campbell, S.H., Fillion, L., Osborne, R.H., \& Maunsell, E. (2015). Measurement invariance of English and French Health Education Impact Questionnaire (heiQ) empowerment scales validated for cancer. Quality of Life Research, 24(10), 2375-2384. doi:10 1007/s11136-015-0972-0

Campbell, S.H., Sanson-Fisher, R., Turner, D., Hayward, L., Wang, X.S., \& Taylor-Brown, J. (2011). Psychometric properties of cancer survivors' unmet needs survey. Supportive Care in Cancer, 19(2), 221-230.

Canadian Cancer Society. (2015). Canadian Cancer Statistics. Toronto, ON: CCS's Steering Committee.

Clausen, C., Strohschein, F.J., Faremo, S., Bateman, D., Posel, N., \& Fleiszer, D.M. (2012). Developing an interprofessional care plan for an older adult woman with breast cancer: From multiple voices to a shared vision. Clinical Journal of Oncology Nursing, 16(1), E18-E25.

Creswell, J.W., \& Plano Clark, V.L. (2011). Designing and conducting mixed methods research. (2 ed.). Thousand Oaks, CA: SAGE Publications.

Curcio, K.R., Lambe, C., Schneider, S.. \& Khan, K. (2012). Evaluation of a cancer survivorship protocol: transitioning patients to survivors. Clinical Journal of Oncology Nursing, 16(4), 400-406.

Daudt, H.M.L., van Mossel, C., Dennis, D.L., Leitz, L., Watson, H.C., \& Tanliao, J.J. (2014). Survivorship care plans: A work in progress. Current Oncology, 21(3), e466-e479.

Dulko, D., Pace, C.M., Dittus, K.L., Sprague, B.L., Pollack, L.A., Hawkins, N.A., \& Geller, B.M. (2013). Barriers and facilitators to implementing cancer survivorship care plans. Oncology Nursing Forum, 40(6), 575-580.

Faul, L.A., Rivers, B., Shibata, D., Townsend, I., Cabrera, P., Quinn, G.P., \& Jacobsen, P.B. (2012). Survivorship care planning in colorectal cancer: Feedback from survivors and providers. Journal of Psychosocial Oncology, 30(2), 198-216.

Feinstein, A.R. (1987). Clinimetrics. New Haven: Yale University Press. Fillion, L., Cook, S., Blais, M-C., Veillette, A-M., Aubin, M., de Serres, M., Rainville, F., ... Fournier, B. (2011). Implementation of screening for distress with professional cancer navigators. Oncologie, 13, 277-289.
Fitch, M.I. (1994). Providing supportive care for individuals living with cancer. Toronto, ON: Ontario Cancer Treatment and Research Foundation.

Forsythe, L.P., Parry, C., Alfano, C.M., Kent, E.E., Leach, C.R., Haggstrom, D.A., ... Rowland, J.H. (2013). Use of survivorship care plans in the United States: Associations with survivorship care. Journal of the National Cancer Institute, 105(20), 1579-1587.

Greimel, E., Lahousen, M., Dorfer, M., Lambauer, M., \& Lang, U. (2011). Patients' view of routine follow-up after gynecological cancer treatment. European Journal of Obstetrics \& Gynecology and Reproductive Biology, 159(1), 180-183.

Grover, S., Hill-Kayser, C., Vachani, C., Hampshire, K., DiLullo, G.A., \& Metz, J.M. (2012). Patient reported late effects of gynecological cancer treatment. Gynecologic Oncology, 124(3), 399-403.

Haq, R., Heus, L., Baker, N.A., Dastur, D., Leung, F-H., Leung, E., Li, B., ... Parsons, J.A. (2013). Designing a multifaceted survivorship care plan to meet the information and communication needs of breast cancer patients and their family physicians: Results of a qualitative pilot study. BMC medical informatics and decision making, 13(76). doi:10.1186/1472-6947-13-76

Hébert, J., \& Fillion, L. (2016). Développement a validation d'un plan de soins de suivi (PSS) pour des femmes atteintes du cancer de l'endomètre lors de la transition de la fin du traitement actifvers la survie au cancer. Manuscrit soumis pour publication.

Hewitt, M., Greenfield, S., \& Stovall, E. (2006). From cancer patient to cancer survivor: Lost in transition. Washington DC: The National Academies Press.

Hill-Kayser, C.E., Vachani, C.C., Hampshire, M.K., Di Lullo, G., Jacobs, L.A., \& Metz, J.M. (2013). Impact of internet-based cancer survivorship care plans on health care lifestyle behaviors. Cancer, 119(21), 3854-3860.

Howell, D., Hack, T.F., Oliver, T.K., Chulak, T., Mayo, S., Aubin, M., Chasen, M., ... Sinclair, S. (2011). Survivorship services for adult cancer population: A Pan-Canadian guideline. Current Oncology, 18(6), e265-e281.

Jefford, M., Lotfi-Jam, K., Baravelli, C., Grogan, S., Rogers, M., Krishnasamy, M., Pezaro, C., ... Schofield, P. (2011). Development and pilot testing of a nurse-led posttreatment support package for bowel cancer survivors. Cancer Nursing, 34(3), E1-E10.

Jones, J.M., Ferguson, S., Edwards, E., Walton, T., McCurdy, N., \& Howell, D. (2012). Experiences of care delivery: Endometrial cancer survivors at end of treatment. Gynecologic Oncology, 124(3), 458-464.

Keesing, S., McNamara, B., \& Rosenwax, L. (2015). Cancer survivors' experience of using survivorship care plans: A systematic review of qualitative studies. Journal of Cancer Survivorship, 9(2), 260-268.

Klemanski, D.L., Browning, K.K., \& Kue, J. (2015). Survivorship care plan preferences of cancer survivors and health care providers: A systematic review and quality appraisal of the evidence. Journal of Cancer Survivorship, 10(1), 71-86. doi:10.1007/s1176401504520

Maunsell, E., Lauzier, S., Brunet, J., Pelletier, S., Osborne, R.H., \& Campbell, H.S. (2014). Health-related empowerment in cancer: Validity of scales from the Health Education Impact Questionnaire. Cancer, 120(20), 3228-3236.

Mayer, D.K., Gerstel, A., Leak, A.N., \& Smith, S.K. (2012). Patient provider preferences for survivorship care plans. Journal of Oncology Practice, 8(4), e80-e86. doi:10.1200/JOP.2011.000401

Mayer, D.K., Nekhlyudov, L., Snyder, C.F., Merrill, J.K., Wollins, D.S., \& Shulman, L.N. (2014). American Society of Clinical Oncology of clinical expert statement on cancer survivorship care planning. Journal of Oncology Practice, 10(6), 345-351. 
McCabe, M.S., \& Jacobs, L.A. (2012). Clinical update: survivorship care-models and programs. Seminars in oncology nursing, 28(3), e1-e8.

McCorkle, R., Ercolano, E., Lazenby, M., Schulman-Green, D., Schilling, L.S., Lorig, K., \& Wagner, E.H. (2011). Self-management: Enabling and empowering patients living with cancer as a chronic illness. Cancer journal for clinicians, 61(1), 50-62.

Meleis, A.I., Sawyer, L.M., Im, E.O., Hilfinger Messias, D.K., \& Schumacher, K. (2000). Experiencing transitions: An emerging middle-range theory. Advances in Nursing Science, 23(1), 12-28.

Miles, M.B., \& Huberman, A.M. (2003). Analyse des données qualitatives. (2nd ed.). Bruxelles: Groupe De Boeck.

Nicolaije, K.A., Husson, O., Ezendam N.P., Vos, M.C., Kruitwagen, R.F., Lybeert, M.L., \& van de Poll-Franse, L.V. (2012). Endometrial cancer survivors are unsatisfied with receive information about diagnosis, treatment and follow-up: A study from the population-based PROFILES registry. Patient Education and Counseling, 88(3), 427-435.

Osborne, R.H., Elsworth, G.R., \& Whitfield, K. (2007). The health Education Impact Questionnaire (heiQ): An outcomes and evaluation measure for patient education and self-management interventions for people with chronic conditions. Patient Education and Counseling, 66(2), 192-201.

Propel Centre for Population Health Impact (2012). Survivors' Unmet Needs Survey-Short Form (French). University of Waterloo, Waterloo, Ontario. Provided by HS Campbell, Principal Investigator, Jan 29, 2013.

Rechis, R., Beckjord, E.B., \& Nutt, S. (2014). Potential benefits of treatment summaries for survivors' health information needs: Results from a Livestrong survey. Journal of Oncology Practice, 10(1), 75-78.

Sabatino, S.A., Thompson, T.D., Smith, J.L., Rowland, J.H., Forsythe, L.P., Pollack, L., \& Hawkins, N.A. (2013). Receipt of cancer treatment summaries and follow-up instructions among adult cancer survivors: Results from a national survey. Journal of Cancer Survivorship: Research and Practice, 7(1), 32-43.

Salani, R. (2013). Survivorship planning in gynecologic cancer patients. Gynecologic Oncology, 130(2), 389-397.

Salz, T., McCabe, M.S., Onstad, E.E., Baxi, S.S., Deming, R.L., Franco, R.A., Glenn, L.A., ... Oeffinger, K.C. (2014). Survivorship care plans: Is there buy-in from community oncology providers? Cancer, 120(5), 722-730. doi:10.1002/cncr.28472
Salz, T., Oeffinger, K., McCabe, M.S., Layne, T.M., \& Bach, P.B. (2012). Survivorship care plans in research and practice. CA: a cancer journal for clinicians, 62(2), 101-117.

Simard, S., \& Savard, J. (2009). Fear of Recurrence Inventory: Development and initial validation of multidimensional measure of fear of cancer recurrence. Supportive care in cancer, 17(3), 241-251.

Simard, S., \& Savard, J. (2015). Screening and comorbidity of clinical levels of fear of cancer recurrence. Journal of Cancer Survivorship: Research and Practice, 9(3), 481-491. doi:10.1007/s1176401504244

Smith, S.L., Singh-Carlson, S., Downie, L., Payeur, N., \& Wai, E.S. (2011). Survivors of breast cancer: Patient perspectives on survivorship care planning. Journal of Cancer Survivorship: Research and Practice, 5(4), 337-344.

Sprague, B.L., Dittus, K.L., Pace, C.M., Dulko, D., Pollack, L.A., Hawkins, N.A., \& Geller, B.M. (2013). Patient satisfaction with breast and colorectal cancer survivorship care plans. Clinical Journal of Oncology Nursing, 17(3), 266-272.

Street, R.L., Makoul, G., Arora, N.K., \& Epstein, R.M. (2009). How does communication heal? Pathway linking clinician-patient communication to health outcomes. Patient Education and Counseling, 74(3), 295-301.

Stricker, C.T., Jacobs, L.A., Risendal, B., Jones, A., Panzer, S., Ganz, P.A., Syrjala, K.L., ... Palmer, S.C. (2011). Survivorship care planning after the Institute of Medicine recommendations: How are we faring? Journal of Cancer Survivorship: Research and Practice, 5(4), 358-370. doi:10.1007/s1176401110964

Stricker, C.T., \& O'Brien, M. (2014). Implementing the commission on cancer standards for survivorship care plans. Clinical Journal of Oncology Nursing, 18(1), 15-22.

Urbaniec, O.A., Collins, K., Denson, L.A., \& Whitford, H.S. (2011). Gynecological cancer survivors: Assessment of psychological distress and unmet supportive care needs. Journal of Psychosocial Oncology, 29(5), 534-551.

Watson, E.K., Sugden, E.M., \& Rose, P.W. (2010). Views of primary care physicians and oncologists on cancer follow-up initiatives in primary care: An online survey. Journal of Cancer Survivorship: Research and Practice, 4(2), 159-166. 